\title{
Nestin expression and clonal analysis of islet-derived epithelial monolayers: insight into nestin-expressing cell heterogeneity and differentiation potential
}

\author{
R Wang ${ }^{1,2}$, J Li ${ }^{1}$, N Yashpal $^{1}$ and $\mathbf{N}$ Gao ${ }^{1}$ \\ ${ }^{1}$ Lawson Health Research Institute, Department of Physiology and Pharmacology, University of Western Ontario, London, Ontario, Canada \\ ${ }^{2}$ Lawson Health Research Institute, Department of Medicine, University of Western Ontario, London, Ontario, Canada \\ (Requests for offprints should be addressed to R Wang; Email: rwang@uwo.ca)
}

\begin{abstract}
There has over the last several years been renewed interest in developing a system for generating new islets and a search for a self-renewing population in the pancreas. In particular, the neural stem cell marker nestin has been implicated as an islet precursor marker and its immunoreactivity has been localized in the islets of Langerhans. This study examines islet-derived epithelial monolayers expanded ex vivo to provide a source of nestin-expressing progenitor cells - a model that will help us understand the role of nestin-expressing cells in islet cell development. When cultured on a type I collagen gel, islets formed confluent monolayers which lacked endocrine phenotypes but were positive for cytokeratin 20 and contained an increased proportion of proliferating nestin-expressing cells, reaching a maximum of $54 \pm 10 \%$. Co-expression
\end{abstract}

studies demonstrated that the nestin-positive cells are heterogeneous, with some nestin-expressing cells colocalizing with the transcription factor PDX-1 and glucose transporter type 2 or lack of co-expression with vimentin. When clonal populations of nestin-positive cells were expanded and subjected to a differentiation protocol, only a population that expressed the transcription factor PDX-1 at the mRNA level was capable of re-expressing insulin at the mRNA and protein level. In conclusion, these studies demonstrate that expanded nestin-expressing cells in vitro from islet-derived epithelial monolayers are heterogeneous; clonal analysis of nestin-positive cells reveals that a distinct subpopulation of nestin/PDX-1-expressing cells is capable of forming insulin-producing cells.

Journal of Endocrinology (2005) 184, 329-339

\section{Introduction}

$\beta$-Cell precursors could theoretically act as a self-renewing and unlimited source of cells; however, their precise phenotypic nature and identifying markers need to be further examined. In recent years, cell populations expressing specific markers of undifferentiated cells such as the protein nestin have been implicated in participation in the neogenesis of newly forming islets. Nestin is a neuronal stem cell marker (Cattaneo \& McKay 1990, Lendahl et al. 1990) which has been shown to generate a wide spectrum of cell types in the developing nervous system, mesenchyme and blood vessels throughout the embryo, and bone marrow cells (Kachinsky et al. 1994, Dahlstrand et al. 1995, Mokry \& Nemecek 1998, 1999, Shih et al. 2001). It is an intermediate filament protein that is involved in cytoskeletal formation as well as cellular rearrangements to facilitate processes characteristic of undifferentiated cells such as migration and mitosis (Palm et al. 2000, Rietze et al. 2001). Its role in differentiation has been well documented in the central nervous system (CNS), where nestin is abundantly expressed in neuro- epithelial stem cells during embryogenesis, but it is nearly absent from all mature CNS cells (Dahlstrand et al. 1995). Furthermore, freshly isolated neural stem cells (nestinpositive), when co-cultured with a muscle line, rapidly differentiate in vitro into myocytes, demonstrating that there is even more plasticity than originally envisaged in the nestin-positive population and that it can contribute to a wide range of cells lineages (Rietze et al. 2001).

Within the pancreas, there has been much evidence of nestin precursor activity, where nestin-positive cells in the pancreas of humans and rats have been described previously. Through the examination of nestin expression patterns, nestin localization has been demonstrated in the pancreatic islets and ducts (Hunziker \& Stein 2000, Street et al. 2004) and nestin expression has been shown during embryogenesis to precede the appearance of $\beta$-cells (Abraham et al. 2002, Huang \& Tang 2003). Street et al. (2004) revealed that in human neonatal pancreatic sections, there are an increased number of islet-associated positive nestin cells as compared with adult islets, suggesting a role for this marker in early pancreatic development. Our own in vivo rat study shows that during embryonic 
development, nestin and insulin frequently co-localize, suggesting that mature $\beta$-cells may derive from a population of cells expressing nestin (Yashpal et al. 2004). Further evidence of the precursor function of nestin has been provided by the ability of islet-derived nestinpositive cells from both fetal and adult pancreas to differentiate ex vivo into pancreatic endocrine, exocrine and hepatic cell phenotypes (Zulewski et al. 2001, Huang \& Tang 2003), that nestin expression was an intermediate step in the differentiation of insulin-producing cells from embryonic stem cells (Lumelsky et al. 2001) and when cultured under certain conditions, selectively expanded nestin-positive cells are capable of generating three dimensional clusters that resemble islet-like clusters with endocrine cell phenotypes (Lumelsky et al. 2001). Furthermore, human-islet-derived cultures enriched for nestinpositive cells express the transporters ABCG2 and MDR-1, showing that these cells have properties that are consistent with a pluripotent stem-cell like population (Lechner et al. 2002).

Controversy exists, however, over the use of nestin as a progenitor marker and its significance in islet neogenesis. Some studies report that nestin immunoreactivity is present in ductal epithelium, a pancreatic compartment that is known to harbor islet progenitors. At another extreme, others have reported that nestin only marks a population of mesenchymal or endothelial cell types, thereby excluding nestin's role in islet cell development (Lardon et al. 2002, Selander \& Edlund 2002, Klein et al. 2003). Such differences in observations may be due to species-specific differences in nestin expression or choice of nestin antibodies used. In light of these observations, our own previous observations in the developing rat pancreas and that of Street et al. (2004) on the human pancreas demonstrate that nestin-positive cells are observed in small clusters of $\beta$-cells randomly distributed through the pancreas - indicating that nestin expression in these extraislet cells is indicative of newly forming islets. Therefore, nestin cannot be completely excluded as a precursor marker and it is important to find a source of nestinpositive cells and to subsequently further evaluate the role of nestin in islet cell neogenesis and development.

Islet cell neogenesis is known to occur through the differentiation of an islet precursor which is present either in the ducts or within the islets. This study aims at expanding a source of nestin-positive cells from the islets of Langerhans, clonally isolating them, and subsequently using a protocol for the differentiation of this population in order to understand the role of nestin-expressing cells in the islet cell development. In view of the recent controversy over nestin-expressing cells, we demonstrate an added dimension in the complexity of this cell type and show that two separate clonally isolated populations can be derived, one which can re-express insulin, and another that cannot, reiterating the heterogeneity of this undifferentiated cell type in culture.

\section{Materials and Methods}

\section{Islet isolation and culture}

Timed pregnant Wistar rats were obtained from Charles River (Quebec, QC, Canada), housed individually under standard conditions and allowed to deliver naturally. All protocols were approved by the Animal Care Committee at the University of Western Ontario, and were in accordance with the guidelines of the Canadian Council on Animal Care. Isolation of the islets was carried on postnatal day 7. Rat pancreata were dissected and subjected to collagenase XI digestion (Sigma), as described previously (Wang et al. 2004). Eight postnatal pancreata were used per isolation and six different islet isolations were performed for each time point in the study. Purification was achieved by a modified Ficoll density gradient (McDaniel et al. 1984). Freshly isolated islets were placed on type I collagen gel and cultured in DMEM/F12 (GIBCO) serum-free medium, as described previously (Wang et al. 2004). The epithelial monolayers were subcultured when the cells had grown to near confluence. Representative islet cultures were examined immediately after isolation (day 0), and the derived epithelial monolayers were examined at 1,2 and 4 weeks, and 2 and 4 months of the culture period using the following investigations.

\section{Clonal nestin-positive cell purification}

The islet-derived epithelial monolayers cultured between 4 and 8 weeks, a culture period during which there was an increased proportion of nestin-positive cells, were dissociated by incubation with $0.025 \%$ trypsin-EDTA. Clonal nestin-positive cells were achieved by hand-picking. Serial hand-picked cells which contained either single cells or two to five clustered cells were placed into 96-well plates and grown on type I collagen with the medium as described above. Once cloned cells grew near confluence, they were subcultured and separated into two groups one group of cells was continually expanded and the other was examined for nestin immunoreactivity using immunofluorescence staining. In all processes, clonal cells which stained positively for nestin were selected for further islet differentiation experimental studies. To date, we have grown six pure nestin-expressing clones.

\section{Immunofluorescence and morphometric analysis}

Freshly isolated islets embedded in 2\% agarose were fixed in $4 \%$ paraformaldehyde, and islet-derived epithelial monolayers and cloned nestin-positive cells were fixed in situ. Cell sections were immunostained with the primary antibodies as follows: mouse anti-rat nestin (Pharmingen, Mississauga, ON, Canada), mouse anti-cytokeratin 20 (CK20; Dako, Mississauga, ON, Canada), guinea pig 
anti-human insulin and rabbit anti-human glucagon (Zymed, Markham, ON, Canada), mouse anti-5-bromo2'-deoxyuridine (BrdU) (Sigma), rabbit anti-telomerase (CalBiochem, San Diego, CA, USA), rabbit anti-glucose transporter type 2 (Glut-2) (Chemicon, Temecula, CA, USA), rabbit anti-PDX-1 (gift from Dr Wright, University of Vanderbilt, Nashville, TN, USA) and rabbit anti-neurogenin 3 (ngn3), a gift from Dr German, University of California, San Francisco, CA, USA), using immunofluorescence, as described previously (Wang et al. 2004, Yashpal et al. 2004). The sections were incubated overnight at $4{ }^{\circ} \mathrm{C}$ with the appropriate dilution of primary antibody. To evaluate cell proliferation, freshly isolated islets and epithelial monolayers were incubated with $10 \mu \mathrm{M}$ BrdU (Sigma), followed by immunostaining for BrdU (Wang et al. 2004). To identify co-expression of phenotypes in the nestin-positive cell populations, double immunofluorescence staining was performed. Fluorescent secondary antibodies labeled with fluorescein isothiocyanate (FITC) or tetramethylrhodamine isothiocyanate (TRITC) were obtained from Jackson Immunoresearch Laboratories (West Grove, PA, USA). Negative controls included the omission of the primary antibodies.

The number of cells expressing nestin and the BrdU labeling index, as well as the co-expression of phenotypes, was determined using a Lecia DMIRE2 inverted microscope connected to computer-assisted Openlab image software (Improvision, Lexington, MA, USA). For each time point, at least 1000 cells were counted, and each time point was repeated six times. The data are expressed as a percentage of the total number of cells counted and compared using a Student's $t$-test, with differences considered to be statistically significant when $P<0 \cdot 05$.

\section{RT-PCR}

Total RNA was extracted from freshly isolated islets, islet-derived epithelial monolayers and cloned nestinpositive cells using the RNAqueous-4 PCR kit (Ambion, Austin, TX, USA). cDNAs were synthesized and PCR assays were performed (Wang et al. 2004). The PCR oligonucleotide primers used to amplify specific cDNA fragments are listed as follows: (i) nestin (rat): $\mathrm{fw}-5^{\prime}-$ TTCCCTTCCCCCTTGCCTAATACC-3'; rv-5'-TGG GCTGAGCTGTTTTCTACTTTT-3' (464 bp), (ii) preproinsulin (human): fw- $5^{\prime}$-TCACACCTGGTGGA AGCTC-3'; rv-5'-ACAATGCCACGCTTCTGC-3' (179 bp), (iii) glucagon: $\mathrm{fw}-5^{\prime}$-CAGAGGAGAACCCC AGATCA-3'; rv-5'-TCATGACGTTTGGCAAGTT-3' (202 bp), (iv) PDX-1: fw-5'-CCACCCCAGTTTACAA GCTC-3'; rv-5'-TGTAGGCAGTACGGGTCCTC-3' (325 bp), (v) Glut-2: fw-5'-TTAGCAACTGGGTCT GCAAT-3'; rv-5'-GGTGTAGTCCTACACTCATG-3' (343 bp), (vi) $\beta$-actin: fw- $5^{\prime}$-GACGGGGTCACCCAC ACTGTGCCCATCTA-3'; rv-5'-CTAGAAGCATTTG CGGTGGACGATGGAGG-3' (660 bp). The amplified products were analyzed on 1\% agarose gels and visualized by ethidium bromide staining. The controls involved omitting reverse transcriptase, cDNA and DNA polymerase and showed no reaction band. Sequencing of preproinsulin PCR products for verification was performed in the Robarts Research Institute Core Molecular Biology Facility (London, ON, Canada).

\section{Differentiation culture and measurement of insulin content}

To determine the potential of nestin-positive cells to differentiate into insulin-expressing cells, clonal nestinpositive cells were subcultured with a defined differentiation medium which contained DMEM:F12 (1:1) (Sigma) medium supplemented with $10 \%$ fetal bovine serum treated with or without $50 \mathrm{ng} / \mathrm{ml}$ keratinocyte growth factor (KGF) (ID Labs, London, ON, Canada) for 1 week (Wang et al. 2004). The epithelial monolayers cultured in a defined differentiation medium served as a control group. After 7 days of differentiation in culture, cells were collected for RT-PCR and morphological analysis. Insulin content and basal insulin release from cloned nestinpositive cells and islet-derived epithelial monolayers after 1 week in differentiation culture were measured using a rat insulin ELISA kit (DRG Diagnostic, Inc., Mountainside, NJ, USA). The insulin content within the cells from each experimental group was determined and normalized to the DNA content and expressed as ng per $\mu$ g DNA (Wang et al. 2004).

\section{Results}

Nestin expression and proliferation in islet-derived epithelial monolayers

Our previous ex vivo examination of the developing rat pancreas (Yashpal et al. 2004) demonstrated that nestinexpressing cells were present during islet development in the fetal and postnatal pancreas, with frequent coexpression with insulin during embryonic life and a high expression level in the islets within the first week of life. We therefore isolated 7-day-old pancreata to examine and promote the expansion of nestin-expressing cells cultured on type I collagen matrix, and found that the staining for the neural stem cell marker nestin increased progressively over the first 4 weeks in vitro (Fig. 1A). Quantitative analysis demonstrated that the percentage of nestinpositive cells at the beginning of the culture period (day 0 ) was $15 \pm 2 \%$ and increased significantly, reaching its peak value of $54 \%$ after 4 weeks of culture $(P<0 \cdot 01$, Fig. 1B). A constant expression of nestin signals at both protein and mRNA levels was maintained in long-term culture as well (Fig. 1C). A highly proliferative capacity was also observed in the epithelial monolayers, with $41 \pm 5 \%$ of the monolayer cells labeling for BrdU after 4 weeks of culture with 
A
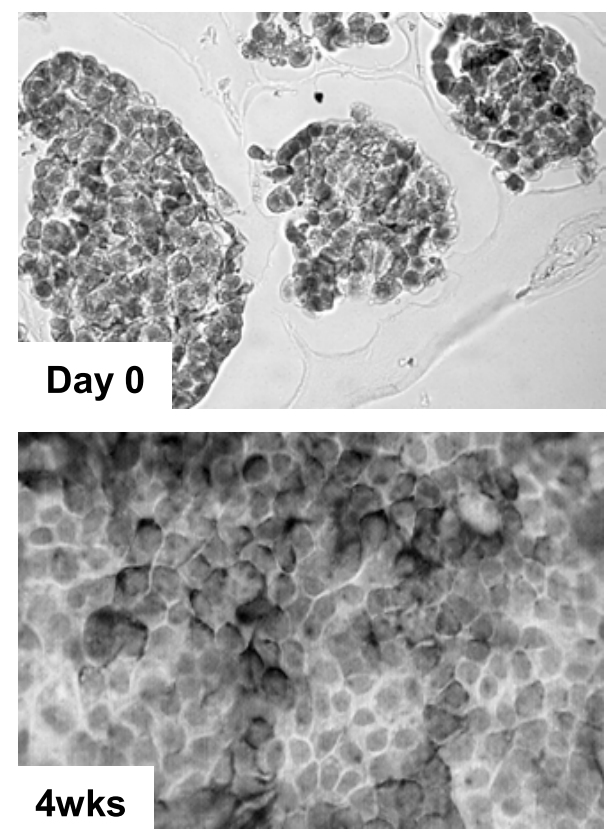

B

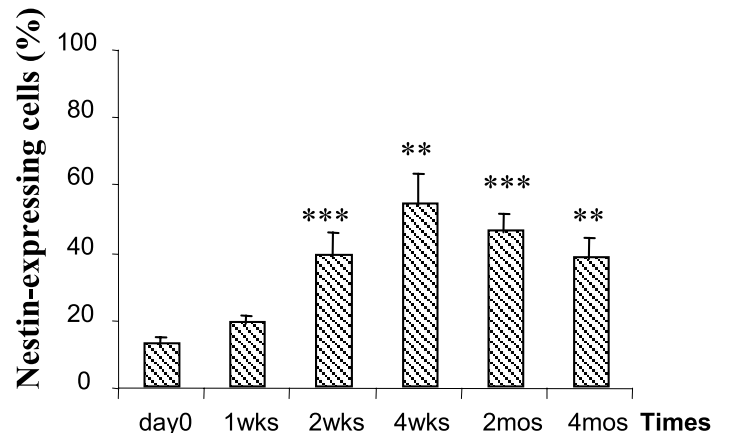

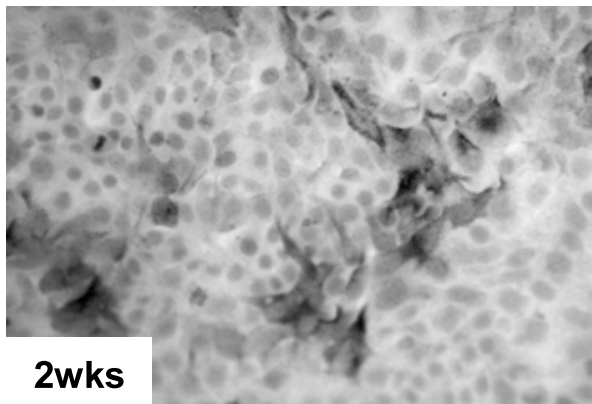

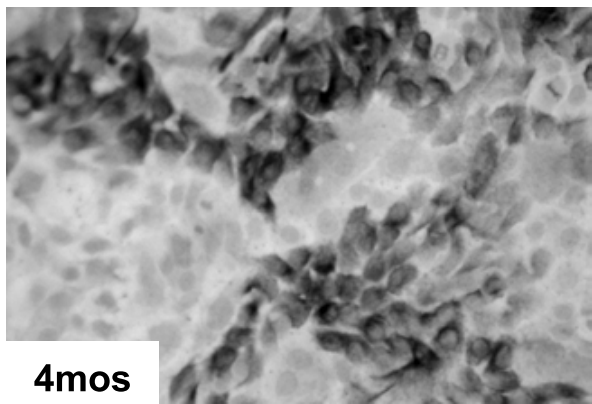

C
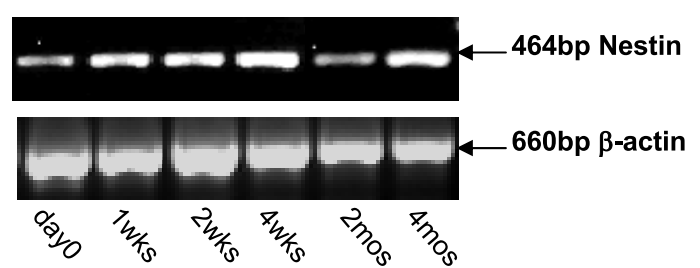

Figure $1 \mathrm{Immunostaining}$ for nestin in freshly isolated islet and islet-derived epithelial monolayers (A) and quantitative analysis of nestin-expressing cells (B) within islet-derived epithelial monolayers during the time of the culture. (C) The expression of nestin mRNA was determined by RT-PCR. ${ }^{* *} P<0 \cdot 01,{ }^{* * *} P<0 \cdot 001$ vs day 0 . Original magnification $\times 400$.

no subsequent significant change in the labeling index after 4 months in vitro (Wang et al. 2004). Double immunostaining for BrdU and nestin at 4 weeks of culture showed that $24 \pm 8 \%$ of nestin-positive cells were BrdU labeled.

Telomerase is an enzyme that can re-lengthen telomeres, providing cells with the ability to proliferate continuously. Figure 2 shows single and double labeling of islet-derived epithelial monolayers using 4,6-diamidino-2phenylindole (DAPI) to stain the nuclei and an antibody against the telomerase catalytic subunit to detect the presence of telomerase. The level of telomerase present in cells during the first 4 weeks of culture was almost undetectable, but frequent telomerase expression was found after 2 months with clear nuclear staining (Fig. 2A). The telomerase signal was located in cells at the edge of the monolayers, which is a region associated with proliferating and migrating cells. Dual immunofluorescent staining for nestin with telomerase (Fig. 2B) indicated that the highly proliferative populations of nestin-positive cells co-expressed telomerase.

Co-expression patterns of nestin-expressing cells in islet-derived epithelial monolayers

To identify co-expression of nestin immunophenotypes in islet-derived epithelial cells, dual immunofluorescent staining was performed. There were two sets of coexpression pattern studies. First co-expression of nestin with transcription factors was examined. Co-staining of nestin with PDX-1 was observed at $15 \pm 4 \%$ in the monolayers after 2 months of culture, while no coexpression of ngn3 and nestin was detected (Fig. 3). Secondly, the co-expression of nestin with Glut-2, a 

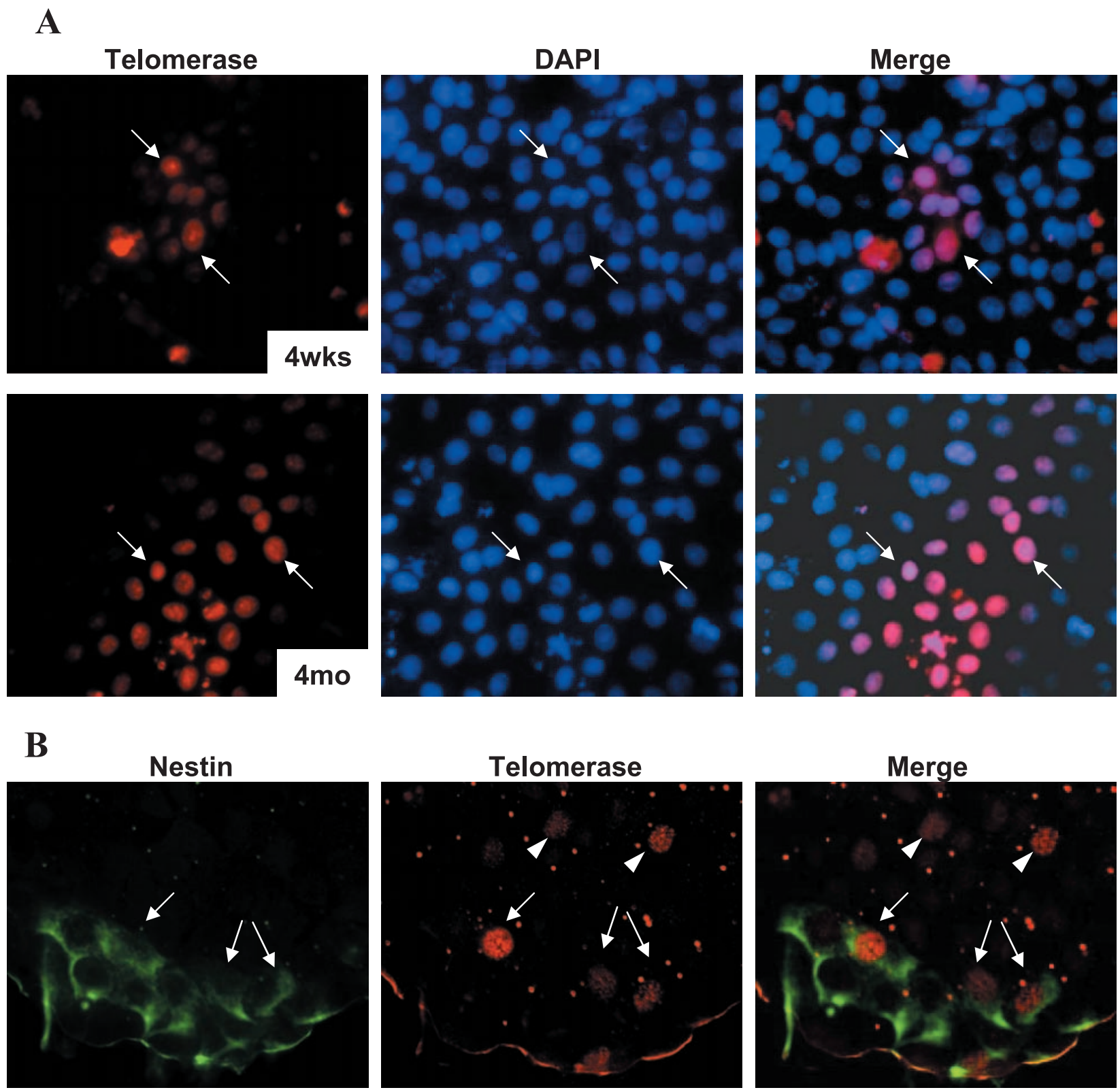

Figure 2 Expression of the telomerase catalytic subunit (A) in islet-derived epithelial monolayers at 4 weeks and 4 months of culture. Cells were stained with anti-telomerase antibody and with DAPI to visualize nuclei; arrows indicate the co-localization. (B) Dual immunofluorescent staining for nestin (FITC) and telomerase (TRITC) in islet-derived epithelial monolayers at 4 months of culture (arrows indicate the co-staining of nestin and telomerase; arrowheads show telomerase-positive nuclei only). Original magnification $\times 400$.

mature $\beta$-cell marker as well as a potential islet cell precursor marker (Pang et al. 1994), and with the mesenchmyal marker vimentin was examined. There were $11 \pm 1 \%$ of nestin-positive cells stained for Glut-2 and the majority of vimentin-positive cells expressed nestin with only $8 \pm 2 \%$ of nestin-positive cells that negatively stained for vimentin in the monolayers after 2 months of culture (Fig. 3).
Cloned nestin-expressing cells and their potential to differentiate

To examine the potential for islet cells to re-form from nestin-positive monolayers in vitro, and more importantly to determine if nestin is a progenitor of insulin-producing cells, a defined differentiation culture medium was utilized to monitor islet cell differentiation. Six cloned 

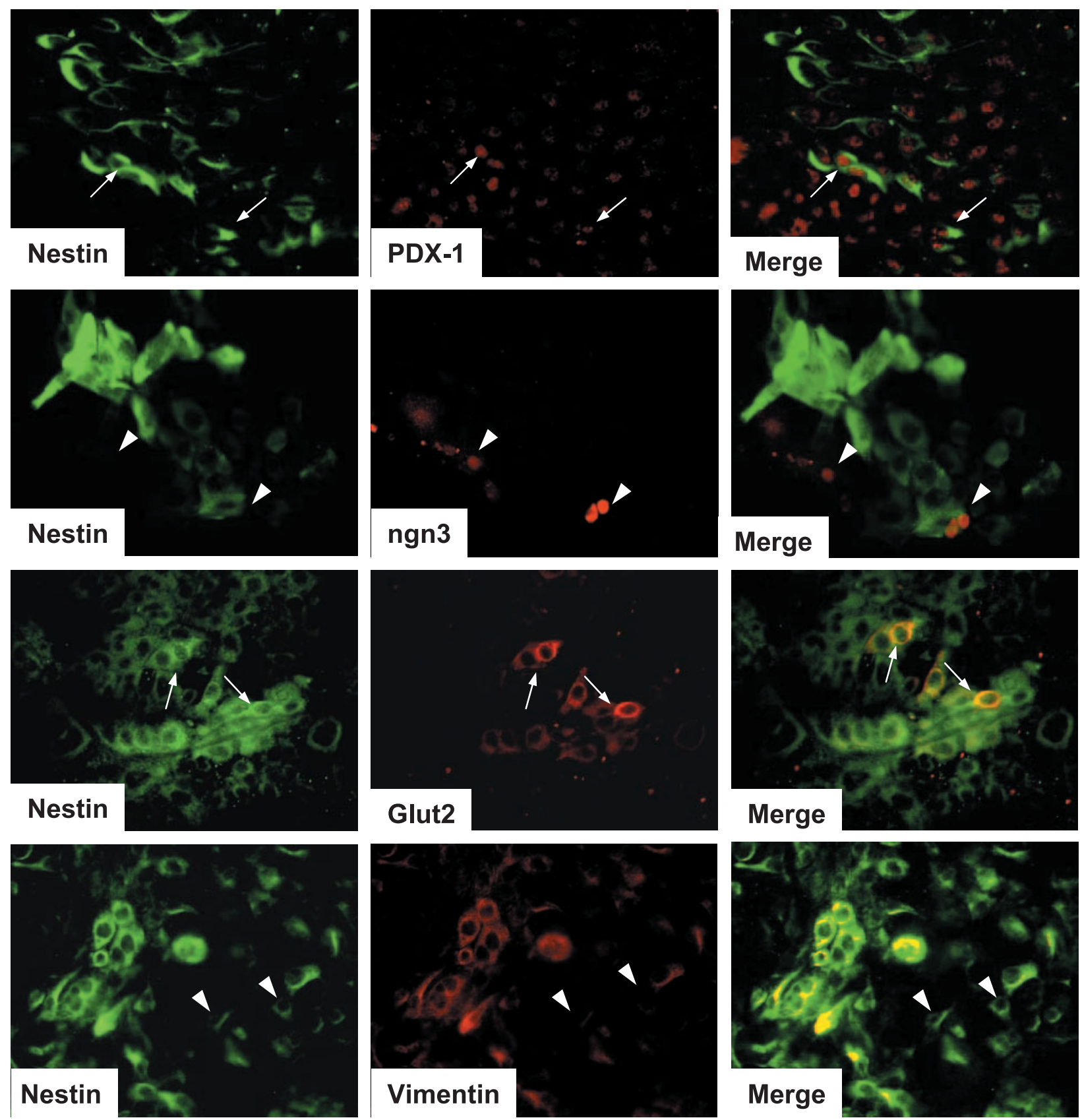

Figure 3 Co-expression of nestin (FITC) with PDX-1, ngn3, Glut-2 and vimentin (TRITC) in islet-derived epithelial monolayers at 2 months of culture determined by double immunofluorescence staining (arrows indicate the co-localization and arrowheads indicate the absent co-staining cells). Original magnification $\times 400$.

nestin-positive cells (Fig. 4A) were subjected to the differentiation treatment. Interestingly, only one (clone number 4017) of the clones showed co-expression of nestin with insulin and insulin with PDX-1 (Fig. 4B and C), while the rest of the clones were negative for either insulin or glucagon as well as PDX-1 staining (Fig. 4D).
RT-PCR analysis demonstrated that clone number 4017 clonally derived nestin-positive cells that co-expressed insulin, also expressed mRNA for the transcription factor PDX-1, as well as preproinsulin and glucagon mRNA (Fig. 5). Those insulin-negative nestin-cloned populations such as clone number 6018 did not express PDX-1 and 
A
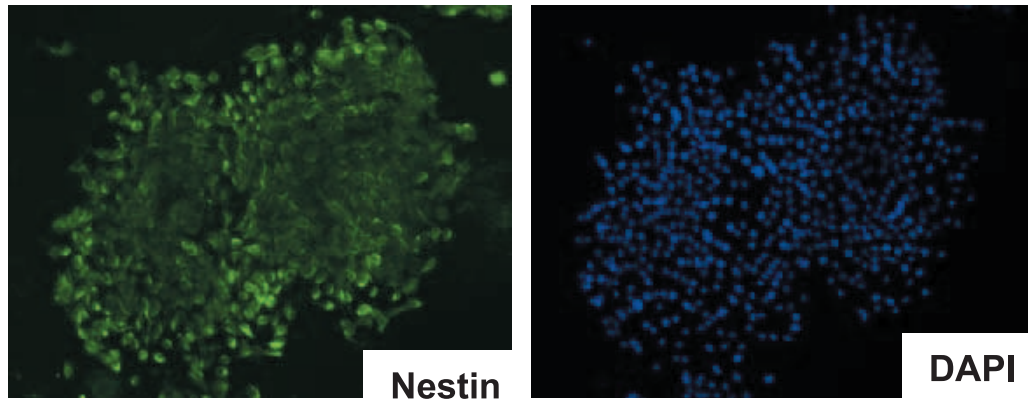

B
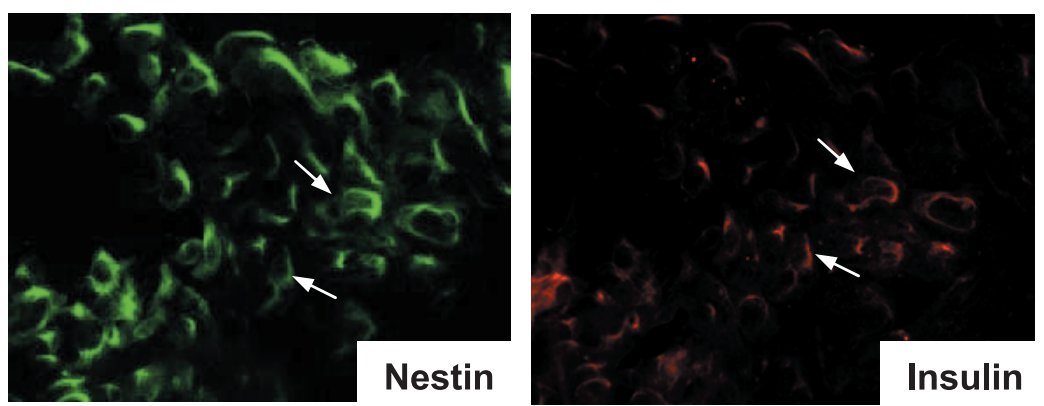

C

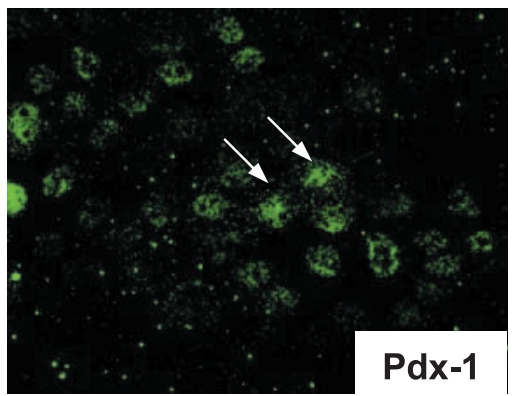

D

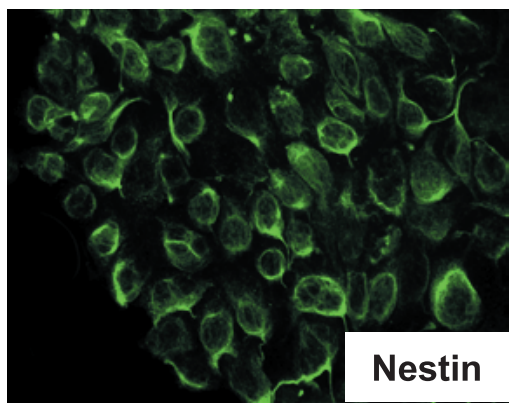

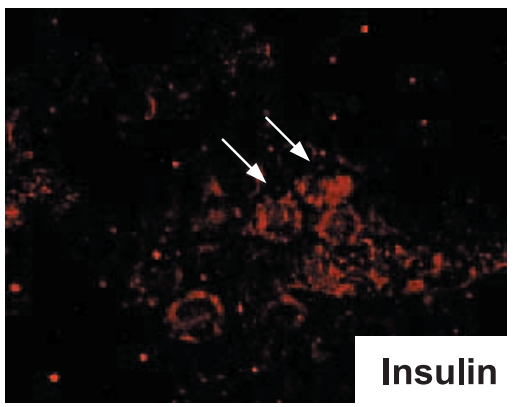

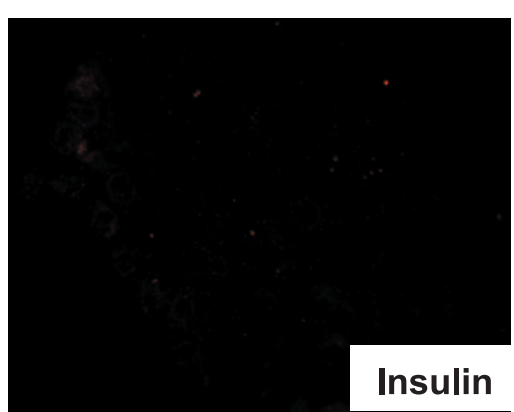

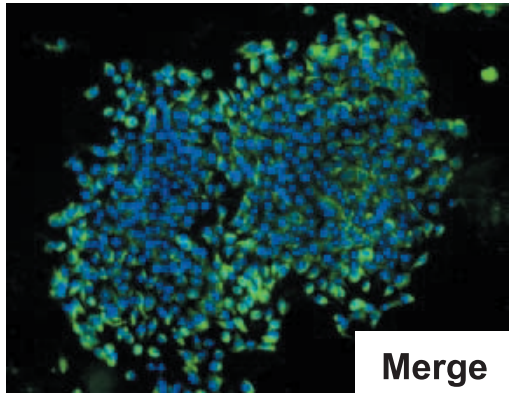
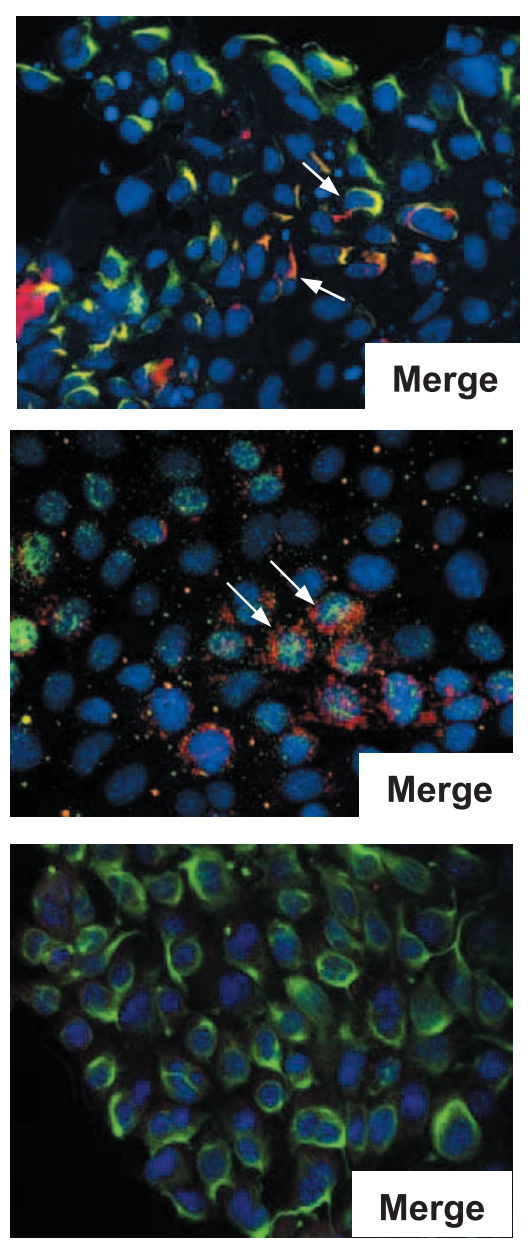

Figure 4 (A) Single nestin-positive cells cloned (clone number 4017) and expanded on type I collagen and immunostained for nestin to determine the purity of the nestin population. Double immunofluorescence for nestin (FITC) with insulin (TRITC) (B), and PDX-1 (FITC) with insulin (TRITC) (C) of clone 4017 was observed during the differentiation culture at 1 week (arrows indicate the co-expression phenotypes), but there is no co-staining of nestin with insulin observed in the rest of the nestin clones (D). Nuclei were counterstained with DAPI. Original magnification $\times 400$.

preproinsulin signals, with only a weak signal for glucagon. Both Glut-2 and nestin mRNA expression was observed in all cloned nestin-positive cells (Fig. 5). However, the signals of preproinsulin and glucagon from clone number 4017 nestin-positive cells and the islet-derived epithelial monolayers were weaker than that of freshly isolated islets, and their cellular insulin content was detected at the lower levels as well (Table 1). In addition, we examined all clones for their expression of the exocrine cell marker amylase after being subjected to the differentiation culture. No amylase mRNA was detected in any of the clones (data not shown). 


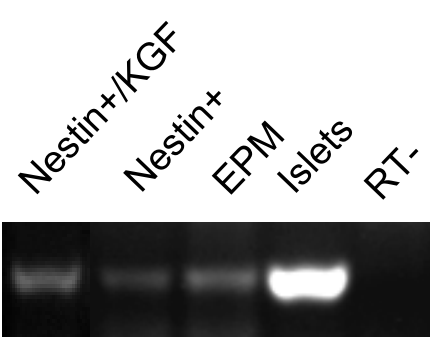

Insulin

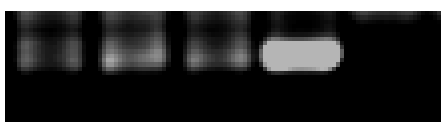

\section{Glucagon}

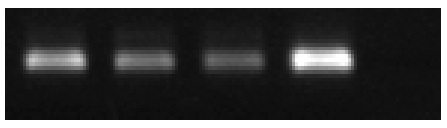

Glut2

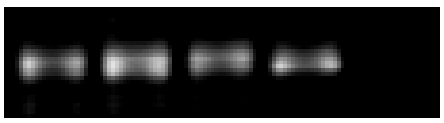

Pdx-1

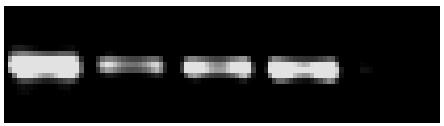

Nestin

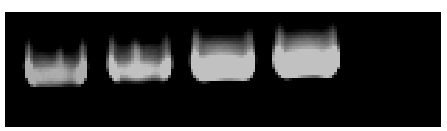

$\beta$-actin

\section{Clone \#4017}
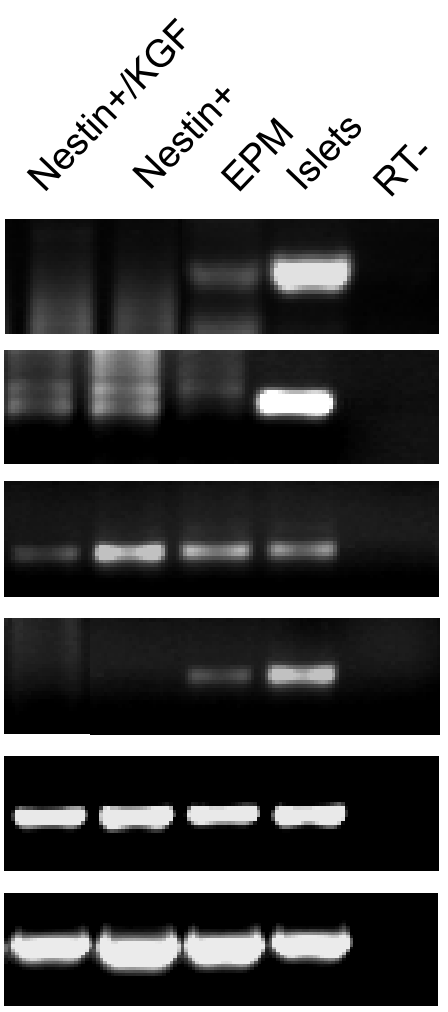

Clone \#6018

Figure 5 RT-PCR analysis of preproinsulin, glucagon, Glut-2, PDX-1 and nestin for cloned nestin-positive cells 4017 and 6018 after 1 week differentiation culture. Nestin +/KGF: differentiation medium contained KGF $(50 \mathrm{ng} / \mathrm{ml})$; Nestin +: only with differentiation medium; EPM: islet-derived epithelial monolayers contain nestin-positive cell populations cultured with differentiation medium; Islets: freshly isolated islets; RT - : omitting reverse transcriptase.

\section{Discussion}

Discovering a pancreatic stem cell is an important step towards creating an unlimited source of islets for transplantation to treat type 1 diabetes. Attention has shifted to nestin and we have characterized the nestin-expressing cells in vitro derived from postnatal rat pancreatic islets. Our study reveals firstly that islets expanded on type I collagen are enriched with populations of nestinexpressing cells that are highly proliferative, can be maintained in long-term culture, and are characterized by their heterogeneous expression of protein markers such as PDX-1, Glut-2 and vimentin. Secondly, we demonstrate that nestin-positive populations can be isolated with distinct gene expression profiles and that only a particular nestin clone that expresses the transcription factor PDX-1

Table 1 Cellular insulin content and basal insulin release of cloned nestin-positive cells and islet-derived epithelial monolayer after 1 week in differentiation culture. Data presented are means \pm S.D. The results were reproduced in triplicate wells

\begin{tabular}{|c|c|c|}
\hline & 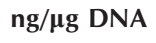 & ng/well \\
\hline \multicolumn{3}{|l|}{ Samples } \\
\hline Nestin-positive/KGF (4017) & $0 \cdot 21 \pm 0 \cdot 10$ & $0 \cdot 28 \pm 0 \cdot 05$ \\
\hline Nestin-positive (4017) & $0 \cdot 19 \pm 0 \cdot 05$ & $0 \cdot 22 \pm 0 \cdot 11$ \\
\hline Islet-derived epithelial monolayers & $0 \cdot 36 \pm 0 \cdot 09$ & $0 \cdot 38 \pm 0.09$ \\
\hline
\end{tabular}


can be induced to differentiate and re-express insulin. Taken together, this study reveals that nestin-expressing cells ex vivo display heterogeneity in their protein and gene expression profiles, and that a distinct fraction of the nestin-positive cells, which are marked through their expression of PDX-1, display endocrine precursor cell activity.

Islets cultured on type I collagen matrix rapidly adhered to the matrix, and spread to form a monolayer of cytokeratin-positive epithelial cells, as described previously (Wang et al. 2001, 2004). Epithelial monolayer formation was preceded by two events, the loss of insulin and glucagon phenotypes, and an increase in cells expressing nestin at both the protein and mRNA level respectively. The population of nestin-expressing cells was maintained at relatively high and constant levels throughout a 4 month culture period as determined by immunocytochemistry and RT-PCR. Our observations also demonstrated that dividing cells persisted within our epithelial monolayers as shown through the incorporation of BrdU. In particular, nestin and BrdU co-localization was also revealed. The observation of nestin and telomerase co-localization is of particular interest. Telomerase is a specific reverse transcriptase that synthesizes new telomeric repeats on the chromosome end to compensate for telomeric loss due to an 'end-replication-problem', providing the basis for an unlimited proliferative capacity (Krupp 2000). It is present in normal immortal human embryonic stem cells (Amit et al. 2000) and in germ line cells, both of which proliferate continuously without signs of aberrant growth control. The identification of nestin and telomerase co-expression suggests further that these nestin-positive cells are undifferentiated, immature cell types.

Analysis of the co-expression patterns of nestin with pancreatic transcription factors and the marker vimentin demonstrated that cells expressing this marker may be heterogeneous. In our previous in vivo examination of nestin expression in the developing rat pancreas, we found that there was a developmental progression of nestin expression that coincides with the formation of newly forming islets. During embryonic to postnatal transition, nestin-positive cells sequentially were characterized by signature protein markers, such that during embryonic life nestin and insulin frequently co-localized, but this occurrence was seldom seen in postnatal life. Also, nestin immunoreactivity was localized to the ducts early in postnatal life but shifted to encircle the ducts, a location of mesenchyme, which was further demonstrated by a population of nestin-expressing cells that co-localized with vimentin-expressing cells. In the present study, such observational findings ex vivo are also observed such that a small proportion of nestin-positive cells were found which expressed the early pancreatic transcription factor PDX-1 and Glut-2. Interestingly, less then 10\% of nestin-expressing cells lack vimentin expression. Thus we concluded that nestin-positive populations are hetero- geneous and may mark not only endocrine progenitors, but also mesenchymal and exocrine cells as well. Furthermore, the population of cells that express PDX-1 and Glut-2 may represent a subpopulation of nestin-positive cells which may play a potential role in islet cell development and retain precursor activity in vitro based on their expression of these early pancreatic progenitor markers.

To address the potential of these nestin-positive cells to differentiate into insulin-expressing cells we isolated nestin-positive cells through a single clone method. Previous studies have documented that nestin expression is an intermediate step in the differentiation of insulinproducing cells (Lumelsky et al. 2001, Yashpal et al. 2004); thus under the appropriate differentiation cues that have been used previously, we examined the potential of our nestin-cloned cells to re-express insulin and glucagon. No islet clusters were formed; however, insulin immunoreactivity and mRNA expression were found in a population of nestin-cloned cells that also expressed the transcription factor PDX-1 at the mRNA level. On the other hand, the majority of the nestin-cloned cell populations did not respond to the differentiation medium and thus lacked insulin or PDX-1 expression. This ex vivo observation reiterates that only a subset of nestinexpressing cells may contribute to newly forming $\beta$-cells. However, it must be noted that the insulin content was lower than that of normal islets, which indicates that adequate $\beta$-cell mass does not form in these cloned nestinpositive/PDX-1 cells. In particular future studies may be conducted to characterize the gene expression profiles, isolate and optimize differentiation of these nestin/ PDX-1-positive cells, as well as the necessary cell-cell interactions and paracrine factors.

Nestin as a marker of pancreatic stem cells has indeed been a pancreatic conundrum. Importantly, no consensus has been reached on the use of nestin as a precursor marker. Although the usefulness of nestin as a precursor marker has been challenged (Lardon et al. 2002, Selander \& Edlund 2002), our own in vivo study, and the works of others, illustrate that nestin expression coincides with the appearance and formation of insulin-producing cells (Lumelsky et al. 2001, Yashpal et al. 2004). Furthermore, nestin-positive cells have been confirmed through in vitro studies to form pancreatic endocrine cell types from adult rat, human and fetal human pancreas (Zulewski et al. 2001, Huang \& Tang 2003). Controversy exists, however, at two levels - from expression pattern studies of nestin and from controversies associated with transgenic studies. First, studies have suggested that nestin is limited to mesenchymal and/or endothelial cells (Lardon et al. 2002, Selander \& Edlund 2002, Klein et al. 2003). Such discrepancies in nestin immunoreactivity, however, may be accounted for by species-specific differences in nestin expression or differences between antibodies used and the heterogeneity of the nestin population. Secondly, the 
debate against nestin as a progenitor marker has been further intensified by many studies that have examined progeny of cells that transcribe the nestin gene by marking them genetically through a Cre/loxP approach (Treutelaar et al. 2003, Delacour et al. 2004, Esni et al. 2004). Despite these elegant transgenic studies the answer to whether or not nestin is a stem cell marker for $\beta$-cells remains a mystery since nestin regulatory elements that are used in several of these studies may be incomplete, Cre expression may not accurately recapitulate the entire pattern of endogenous nestin expression or because nestin-positive cells from which endocrine cells arise are from a fraction of nestin-positive cells in which expression results from cis-regulatory elements not present in the transgenes used. Through Cre-mediated labeling, Esni et al. (2004) demonstrated that pancreatic exocrine cells in the developing pancreas originate from nestin-positive cells and that PDX-1 co-expressed with nestin in pancreatic epithelium. However, they were unable to exclude the expression of nestin in an early endocrine precursor. They concluded that there may be a low frequency of endocrine differentiation within the broader population of nestin-positive and ngn3-positive cells. Interestingly, in our own study, a very small proportion of nestin-cloned cells co-expressed the transcription factor PDX-1 and could differentiate to re-express insulin, suggesting that there may very well be a small subpopulation of nestin-positive cells with the potential to give rise to new endocrine cells, while none of the clones expressed the exocrine marker amylase. More recently, explants of neogenic ductules isolated from rats after partial pancreatectomy were characterized by their expression of nestin-positive ductal stem cells, which were found adjacent to newly forming single endocrine cells or small clusters (Kim et al. 2004). Importantly, a subpopulation of nestin-positive cells expressed PDX-1, a finding that is interesting in the context of our own study. These findings and our own only further suggest that nestinexpressing PDX-1-positive cells may in fact represent a cell population capable of cytodifferentiation into insulinsecreting cells.

In conclusion we have shown the presence of a subset of nestin-positive cells in vitro that under the appropriate environmental cues can differentiate and re-express insulin. The data presented strongly suggest that nestin is definitely a heterogeneous cell marker and that although the majority of nestin-positive cells in adults may not be islet precursors, there still remains a population that requires further investigation and examination. In particular the analysis and comparison of nestin clones should be closely examined and our study highlights that nestinpositive populations that express PDX-1 with the ability to re-express insulin are a population that must be examined and exploited. The involvement of nestin in islet cell neogenesis cannot be ruled out without an exhaustive assessment of all the nestin-positive populations expressing the protein. Isolation and clonal analysis of nestin-positive cells will provide further insight into the cellular role of nestin in islet cell development and differentiation.

\section{Acknowledgements}

This work was supported by grants from the Canadian Institute of Health Research. R W is supported by a University Faculty Award from the Natural Science \& Engineering Research Council of Canada. The authors declare that there is no conflict of interest that would prejudice the impartiality of this scientific work.

\section{References}

Abraham EJ, Leech CA, Lin JC, Zulewski H \& Habener JF 2002 Insulinotropic hormone glucagon-like peptide-1 differentiation of human pancreatic islet-derived progenitor cells into insulin-producing cells. Endocrinology 143 3152-3161.

Amit M, Carpenter MK, Inokuma MS, Chiu CP, Harris CP, Waknitz MA, Itskovitz-Eldor J \& Thomson JA 2000 Clonally derived human embryonic stem cell lines maintain pluripotency and proliferative potential for prolonged periods of culture. Developmental Biology 277 271-275.

Cattaneo E \& McKay R 1990 Proliferation and differentiation of neuronal stem cells regulated by nerve growth factor. Nature $\mathbf{3 4 7}$ $762-765$

Dahlstrand J, Lardelli M \& Lendahl U 1995 Nestin mRNA expression correlates with the central nervous system progenitor cell state in many, but not all regions of the developing central nervous system. Developmental Brain Research 84 109-129.

Delacour A, Nepote V, Trumpp A \& Herrera PL 2004 Nestin expression in pancreatic cell lineages. Mechanisms of Development 121 3-14.

Esni F, Stoffers DA, Takeuchi T \& Leach SD 2004 Origin of exocrine pancreatic cells from nestin-positive precursors in developing mouse pancreas. Mechanisms of Development 121 15-25.

Huang H \& Tang X 2003 Phenotypic determination and characterization of nestin-positive precursors derived from human fetal pancreas. Laboratory Investigation 83 539-547.

Hunziker E \& Stein M 2000 Nestin-expressing cell in the pancreatic islets of Langerhans. Biochemical and Biophysical Research Communications 271 116-119.

Kachinsky AM, Dominov JA \& Miller JB 1994 Myogenesis and the intermediate filament protein, nestin. Developmental Biology 165 216-228.

Kim SY, Lee SH, Kim BM, Min EH, Bendayan M \& Park IS 2004 Activation of nestin-positive duct stem cells in pancreas upon neogenic motivation and possible cytodifferentiation into insulinsecreting cells from NPDS cells. Developmental Dynamics 230 1-11.

Klein T, Ling Z, Heimberg H, Madsen OD, Heller RS \& Serup P 2003 Nestin is expressed in vascular endothelial cells in the adult human pancreas. Journal of Histochemistry and Cytochemistry $\mathbf{5 1}$ 697-706.

Krupp G 2000 Cell proliferation, carcinogenesis and diverse mechanisms of telomerase regulation. Cell and Molecular Life Sciences 57 464-486.

Lardon J, Rooman I. \& Bouwens L 2002 Nestin expression in pancreatic stellate and angiogenic endothelial cells. Histochemistry and Cell Research 117 535-540.

Lechner A, Leech CA, Abraham EJ, Nolan AL \& Habener JF 2002 Nestin-positive progenitor cells derived from adult human pancreatic islets of Langerhans contain side population (SP) cells 
defined by expression of the ABCG2 (BCRP1) ATP-binding cassette transporter. Biochemical and Biophysical Research Communications 293 670-674.

Lendahl G, Zimmerman LB \& McKay RD 1990 CNS stem cells express a new class of intermediate filament protein. Cell $\mathbf{6 0}$ 585-595.

Lumelsky N, Blondel O, Laeng P, Velasco I., Ravin R \& McKay R 2001 Differentiation of embryonic stem cells to insulin-secreting structures similar to pancreatic islets. Science 292 1389-1394.

McDaniel ML, Colca JR \& Kitagal M 1984 Islet cell isolation and characterization. In Methods in Diabetes Research, vol 1 Laboratory Methods, pp 153-166. Eds J Larner \& S Pohl. New York, NY: John Wiley.

Mokry J \& Nemecek S 1998 Angiogenesis of extra- and intraembryonic blood vessels is associated with expression of nestin in endothelial cells. Folia Biologica (Praha) 44 155-161.

Mokry J \& Nemecek S 1999 Cerebral angiogenesis shows nestin expression in endothelial cells. General Physiology and Biophysics Suppl 11 25-29.

Palm K, Salin-Nordstrom T, Levesque MF \& Neuman T 2000 Fetal and adult human CNS stem cells have similar molecular characteristics and developmental potential. Developmental Brain Research 78 192-195.

Pang K, Mukonoweshuro C \& Wong GG 1994 Beta cells arise from glucose transport type 2 (Glut-2)-expressing epithelial cells of the developing rat pancreas. PNAS 91 9559-9563.

Rietze RL, Valcanis H, Brooker GF, Thomas T, Voss AK \& Bartlett PF 2001 Purification of a pluripotent neural stem cell from the adult mouse brain. Nature 412 736-739.

Selander L \& Edlund H 2002 Nestin is expressed in mesenchymal and not epithelial cells of the developing mouse pancreas. Mechanisms of Development 113 189-192.
Shih CC, Weng Y, Mamelak A, LeBon T, Hu MC \& Forman SJ 2001 Identification of a candidate human neurohematopoietic stem-cell population. Blood 98 2412-2422.

Street CN, Lakey JR, Seeberger K, Helms L, Rajotte RV, Shapiro AM \& Korbutt GS 2004 Heterogeneous expression of nestin in human pancreatic tissue precludes its use as an islet precursor marker. Journal of Endocrinology 180 213-225.

Treutelaar MK, Skidmore JM, Dias-Leme CL, Zhang L, Simeone D, Martin DM \& Burant CF 2003 Nestin-lineage cells contribute to the microvasculature but not endocrine cells of the islet. Diabetes $\mathbf{5 2}$ 2503-2512.

Wang R, Li J \& Rosenberg L 2001 Factors mediating the transdifferentiation of islets of Langerhans to duct epithelial-like structures. Journal of Endocrinology 171 309-319.

Wang R, Li J \& Yashpal N 2004 Phenotypic analysis of c-Kit expression in epithelial monolayer derived from postnatal rat pancreatic islets. Journal of Endocrinology 182 113-122.

Yashpal N, Li J \& Wang R 2004 Characterization of c-Kit and nestin expression during islet cell development in the prenatal and postnatal rat pancreas. Developmental Dynamics 229 813-825.

Zulewski H, Abraham EJ, Gerlach MJ, Daniel PB, Moritz W, Muller B, Vallejo M, Thomas MK \& Habener JF 2001 Multipotential nestin-positive stem cells isolated from adult pancreatic islets differentiate ex vivo into pancreatic endocrine, exocrine, and hepatic phenotypes. Diabetes 50 521-533.

Received 13 October 2004

Accepted 2 November 2004

Made available online as an

Accepted Preprint 15 November 2004 